\title{
Pilot Study, The First Step in Research
}

\author{
Ronald E. Mocorro \\ Leyte Normal University, Philippines, Tacloban City, Philippines 6500
}

\begin{abstract}
A pilot study is very important in the success of the research. It is often recommended to address variety of issues such as the validity and reliability of the instrument to be used in the study. This article investigates how often graduate students researches employ pilot study. It also investigates their discussion of the result as to partial or complete discussion of the result of the pilot study. Specific recommendations are made to the researchers in employing pilot study before the conduct of the full research.
\end{abstract}

Keywords: Pilot study, Pilot testing, Pilot test, Development of questionnaire

\section{Introduction}

The validity and reliability of the instrument or questionnaire are some of the important issues to consider in conducting research. Often, these can be seen by analyzing the result of the pilot study. The reliability and validity of the instrument are vital for the future result of the research. If the instrument is not reliable and not valid, then the result conducted is void.

According to the Association for Qualitative Research, a pilot study is a small study conducted in advance of a planned project, specifically to test aspects of the research design and to allow necessary adjustment before final commitment to the design. Similarly, pilot studies are defined as a mini version of a full-scale study which is also known as feasibility studies. It is also defined as the process of pre-testing of the research instrument such as questionnaire, tests, and interview schedule [6], [7].

Conducting pilot study has several advantages; it gives advance warning about where the main project fail, which research procedure where not followed, or whether the proposed methods or the questionnaire or instrument are inappropriate or too complicated to the respondents [7].

In the words of De Vaus "Do not take the risk. Pilot test first." [3]. These are important reasons for undertaking a pilot study, but there are additional reasons, for example convincing funding bodies that your research proposal for the main study is worth funding. Thus pilot studies are conducted for a range of different reasons. Table 1 enumerates the reasons for conducting pilot studies.

Welman and Krunge[8] added that the following are the main reason why the pilot study is needed:

- Detect possible flaws in measurement procedures. This includes instructions, administration, time limits, etc.

- Identify unclear or ambiguous items in the questionnaire.

- Identify non-verbal behavior of the participants which may affect research study. This may give important information about any embarrassment or discomfort experienced concerning the content or wording of items in a questionnaire.

\section{Table 1. Reason why pilot studies should be} conducted

- Developing and testing adequacy of research instruments

- Assessing the feasibility of a (full-scale) study/survey

- Designing a research protocol

- Assessing whether the research protocol is realistic and workable

- Establishing whether the sampling frame and technique are effective

- Assessing the likely success of proposed recruitment approaches

- Identifying logistical problems which might occur using proposed methods

- Estimating variability in outcomes to help to determine sample size

- Collecting preliminary data

- Determining what resources (finance, staff) are needed for a planned study

- Assessing the proposed data analysis techniques to uncover potential problems

- Developing a research question and research plan

- Training a researcher in as many elements of the research process as possible

- Convincing funding bodies that the research team is competent and knowledgeable

- Convincing funding bodies that the main study is feasible and worth funding

- Convincing other stakeholders that the main study is worth supporting

A Pilot study is crucial to the success of the research. It should be done correctly so that it will give a correct description of the instrument to be used. The validity and reliability should be established. Here are the steps used to pilot a questionnaire on a small group of volunteers, who are as similar as possible to the target population, are listed in Table 2.

Table 2: Pilot study procedures to improve the internal validity of a questionnaire

- administer the questionnaire to pilot subjects in exactly the same way as it will be administered in the main study

- $\quad$ ask the subjects for feedback to identify ambiguities and difficult questions

- record the time taken to complete the questionnaire and decide whether it is reasonable

Volume 6 Issue 12, December 2017 


\section{International Journal of Science and Research (IJSR) \\ ISSN (Online): 2319-7064}

Index Copernicus Value (2016): 79.57 | Impact Factor (2015): 6.391

- discard all unnecessary, difficult or ambiguous questions

- assess whether each question gives an adequate range of responses

- establish that replies can be interpreted in terms of the information that is required

- check that all questions are answered

- re-word or re-scale any questions that are not answered as expected

- shorten, revise and, if possible, pilot again.

(Source: Table 3.23 in Peat et al., 2002: 123)

\section{Objectives}

Pilot study is often recommended before the actual study. This is a preliminary trial of research which is essential in the whole research process. The purpose of this study is to examine thesis and dissertations of the graduate's students to answer the following questions;

1) How often graduate students researches utilize pilot studies?

2) How often researchers discussed the result of the pilot study in the research paper?

\section{Methodology}

The study employed summative content analysis of 35 total enumerated thesis and dissertation in the graduate school of one university in the region. A summative content analysis involves counting and comparisons, usually of keywords or content, followed by the interpretation of the underlying context [4]. The researcher utilizes total enumeration or use all the thesis/dissertation that can be found at the graduate school library of the said university from the school year 2011- 2017. Frequency counts and percent were used to analyze the data.

\section{Result}

Table 3: Percentage of thesis / dissertations employed pilot study

\begin{tabular}{|l|c|c|}
\hline & f & $\%$ \\
\hline Employed Pilot Study & 12 & $34 \%$ \\
\hline Did not Employ Pilot Study & 23 & $66 \%$ \\
\hline Total & 35 & $100 \%$ \\
\hline
\end{tabular}

Table 3 shows that among the total of 35 researches, it shows that only 12 which is $34 \%$ of them employed pilot study and the remaining 23 or $66 \%$ did not employ pilot study of their instrument.

Table 4: Employed discussion of pilot study

\begin{tabular}{|c|c|c|c|}
\hline & f & $\%$ & $\%$ ( out of total researches) \\
\hline Partially discussed the & 8 & $67 \%$ & $23 \%$ \\
\hline Completely discussed & 4 & $33 \%$ & $11 \%$ \\
\hline Total & 35 & $100 \%$ & $34 \%$ \\
\hline
\end{tabular}

From table 4 , it displays that only 4 or $33 \%$ of those who employed pilot study includes complete discussions of the result. The results include ways in validating the questionnaire, establishing the reliability by providing the reliability coefficient. Among these, only one researcher expanded the result and use it to further improve the instrument (e.g. revision or removal of poor items in the test/questionnaire). In general, only $11 \%$ of thesis/dissertations completely discussed the result of the pilot study and only a very small percentage include the discussion in improving the instrument or questionnaire. However, $23 \%$ of the said sample partially discussed the findings of the pilot study. Partially meaning it lacks discussion on the process of pilot study as well as the coefficient/ index that describe the result or findings. For example, a study stated that a pilot testing was done to establish the validity and reliability of the questionnaire, however this is not enough since no discussion of the result and thus readers may not be convinced if truly the validity and reliability of the questionnaire were established.

Table 5: Research instrument used

\begin{tabular}{|c|c|c|c|}
\hline & $\mathrm{F}$ & $\%$ & Employed Pilot study \\
\hline Enhanced/modifies/ adapted & 14 & $40 \%$ & 1 \\
\hline Self-made / researcher - structured & 17 & $49 \%$ & 9 \\
\hline Fully utilized & 4 & $11 \%$ & 0 \\
\hline Total & 35 & $100 \%$ & \\
\hline
\end{tabular}

Table 5 shows that 14 or $40 \%$ of the instruments or questionnaires were adapted, enhanced or modified from foreign authors. This means that the researchers made some revision of the items to suit to the locale, such as the language, culture, etc. 17 or $49 \%$ of the questionnaires/ instruments were self-made or researcher- structured instrument. The remaining 4 instrument or about $11 \%$ are fully utilized instrument from published researchers with local authors.

Table shows that only 1 out of 9 adapted or enhanced instrument were pilot tested, 9 out of 17 self-made or researcher-structured instrument were piloted and none from fully utilized instrument.

\section{Discussion}

A Pilot study is very important to establish the validity and reliability of the research question. The result of the pilot study gives the researcher ideas on how to improve the questionnaire or instrument so that the result for study or research will also be reliable and valid. Unfortunately, the study shows that most researchers did not employ pilot study. For some, they employed pilot study but fail to discuss completely the result. It left the reader questioning the validity and reliability of the instrument since no explanations were given. And, very few discussed completely the result of their pilot study.

\section{Conclusion and Recommendations}

A Pilot study is very much important in the success of the research. It identifies variety of issues about the instrument and the research in general. Therefore, it should be taken with almost consideration. Pilot study should be carefully planned and analyzed specially if the researcher uses researcher-made instrument. This instrument is subjected to determine its validity and reliability. No research-made questionnaire should be used to conduct research without it undergone pilot study. Adapted, modified or enhanced instrument should also be pilot tested especially if it is

\section{Volume 6 Issue 12, December 2017}




\section{International Journal of Science and Research (IJSR) \\ ISSN (Online): 2319-7064}

Index Copernicus Value (2016): 79.57 | Impact Factor (2015): 6.391

adapted from foreign authors. The diversity of the respondents, the culture, language, the age, the economic status and many more are factors that made group of individuals differ. As an example a questionnaire that asks about physical activity and uses skiing as an example may not be relevant in settings where there is no snow.

Adaptation of instrument is a complex process and require careful planning to maintain its psychometric properties [2]. It is also important that the adaptation of an instrument should culturally fit, that is, its preparation for use in different cultural contexts [1]. A Pilot study should be done to check and verify that it possesses the psychometric properties and it fit to the cultural contexts. Therefore, the psychometric properties of the new version of instrument should be established and the semantic equivalence of the items should also be shown.

\section{References}

[1] Beaton DE, Bombardier C, Guillemin F, Ferraz MB: Guidelines for the process of cross-cultural adaptation of self-report measures. Spine. 2000, 25

[2] Cassepp-Borges, V., Balbinotti, M. A. A., \&Teodoro, M. L. M. (2010). Tradução e validação de conteúdo: Uma proposta para a adaptação de instrumentos. In L. Pasquali, Instrumentaçãopsicológica: Fundamentos $e$ práticas(pp. 506-520)

[3] De Vaus D (1993). Survey in Social Research. Third edition. London, UCL Press.

[4] Hsiu-Fang Hsieh, Sarah E. Shannon (2005). Three Approaches of Qualitative Content Analysis. Qualitative Health Research. Sage Journal

[5] International Test Commission [ITC], 2010

[6] Polit D et al (2001). Essential of Nursing Research. Methods Appraisal and Utilization. Fifth edition. Philadelphia PA. Lippincott William and Wilkins.

[7] Van Teijlingen et al, (2001). The importance of conducting and reporting pilot studies; Journal of Advance Nursing, 34, 3, 289-295.

[8] Welman and Krunger( 1999). Research methodology for the business and administrative sciences. Oxford: Oxford University Press

\section{Author Profile}

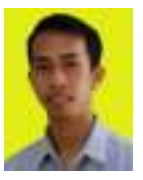

The Author received his B.S, Degree (Bachelor in Elementary Education - Bachelor in Secondary Education- Major in Mathematics, 2009) from Leyte Normal University, Philippines and Master of Mathematics from University of San Carlos, Cebu,

Philippines (2012).

Volume 6 Issue 12, December 2017

www.ijsr.net

Licensed Under Creative Commons Attribution CC BY 